Pacific Journal of Mathematic 


\section{A CUSHIONING-TYPE WEAK COVERING PROPERTY}

\section{S. W. DAVIS}

We discuss certain covering properties which are based on Michael's notion of a cushioned collection. In particular, we discuss property $L$ of Bacon and certain variations on property $L$ in connection with isocompactness and the relationship between $\aleph_{1}$-compactness and the Lindelöf property. We then introduce property $\theta L$ which is a common generalization of property $L$ and weak $\delta \theta$-refinability, and consider uses of this property in similar connections.

o. Introduction. There are a large number of covering properties generalizing paracompactness which have been the subjects of investigation in the last thirty years. Most of these involve some sort of generalization of the notion of locally finite refinements. For example, the metacompact, meta-Lindelöf, $\theta$-refinable, weakly $\theta$-refinable, $\delta \theta$-refinable and weakly $\delta \theta$-refinable spaces are all classes defined by covering properties of this type.

In 1970, Bacon presented a covering property, property $L$, which is based on a generalization of Michael's characterization of paracompactness in terms of cushioned refinements. We present certain variations on property $L$ and discuss how these may be used as hypotheses in place of weak $\delta \theta$-refinability in a number of theorems.

We present a new covering property, property $\theta L$, which is a common generalization of property $L$ and weak $\delta \theta$-refinability and still implies isocompactness [every closed countably compact subset is compact]. We discuss certain variations on property $\theta L$, and find that many of the results obtained using property $L$ or weak $\delta \theta$ refinability remain true when one of these variations is used in place of property $L$ or weak $\delta \theta$-refinability in the hypothesis. In particular, we establish a number of results relating $\aleph_{1}$-compactness with the Lindelöf property and with closed completeness.

We now list certain conventions which will be used in this paper. A perfect mapping is a closed continuous function with the property that the inverse image of each point in the range is compact. We indicate a function $f$ whose domain is the set $A$ and whose range is contained in the set $B$ by $f: A \rightarrow B$. For a collection $\mathscr{A}$ of sets, $\cup \mathscr{A}=U\{A: A \in \mathscr{A}\}$ and ord $(x, \mathscr{A})=|\{A: x \in A \in \mathscr{A}\}|$. If $f: X \rightarrow Y$ and $A \subset X$, we write $f(A)$ to indicate the set $\{f(x): x \in A\}$.

We include for the benefit of the reader the following definitions. Original sources are listed in [16].

Definition 0.1. Suppose $X$ is a space and $\mathscr{W}^{-}$is an open cover 
of $X$ of the form $\mathscr{W}^{-}=\bigcup_{n \in \omega} \mathscr{V}_{n}$. Consider the following conditions on $\mathscr{W}$ :

(i) For each $n \in \omega$, $\mathscr{C}_{n}$ covers $X$.

(ii) For each $x \in X$, there exists $n_{x} \in \omega$ such that $0<$ ord $\left(x, \mathscr{\mathscr { N }}_{n_{x}}\right)<\aleph_{0}$.

(iii) For each $x \in X$, there exists $n_{x} \in \omega$ such that $0<$ ord $\left(x, \mathscr{Y}_{n_{x}}\right) \leqq \aleph_{0}$.

(A) If every open cover of $X$ has a refinement $\mathscr{W}$ satisfying (iii), then $X$ is called weakly $\delta \theta-r e f i n a b l e$.

(B) If every open cover of $X$ has a refinement $\mathscr{W}$ satisfying (i) and (iii), then $X$ is called $\delta \theta$-refinable.

(C) If every open cover of $X$ has a refinement $\mathscr{W}^{-}$satisfying (ii), then $X$ is called weakly $\theta$-refinable.

(D) If every open cover of $X$ has a refinement $\mathscr{W}$ satisfying (i) and (ii), then $X$ is called $\theta$-refinable.

1. Property L. In [12], Michael characterized paracompactness in terms of open covers having cushioned refinements. In this section, we present a family of covering properties which generalize paracompactness using a generalization of the notion of a cushioned refinement. We denote by Card the class of all infinite cardinal numbers.

Definition 1.1. For $\kappa \in$ Card, and $\mathscr{C}$ and $\mathscr{V}$ collections of subsets of a space $X$, we say $\mathscr{V}$ is $\kappa$-weakly cushioned in $\mathscr{U}$ if and only if there exists a function $f: \mathscr{V} \rightarrow \mathscr{W}$ such that if $\mathscr{G} \subset \mathscr{V}$ with $|\mathscr{G}| \leqq \kappa$ and $x: \mathscr{G} \rightarrow \cup \mathscr{G}$ with $x(G) \in G$ for each $G \in \mathscr{G}$, then $\overline{\{x(G): G \in \mathscr{G}\}} \subset \cup f(\mathscr{G})$.

We say a space $X$ satisfies property $\kappa L$ if and only if for every open cover $\mathscr{U}$ of $X$ there is a sequence $\left\langle\mathscr{D}_{n}: n \in \omega\right\rangle$ of collections of subsets of $X$ such that $\bigcup_{n \in \omega} \mathscr{D}_{n}$ is a covering of $X$, and for each $n \in \omega, \mathscr{D}_{n}$ is $\kappa$-weakly cushioned in $\omega \mathscr{\mathscr { C }}$. ( $\omega \mathscr{\mathscr { C }}$ is the set of unions of countable subcollections of $\mathscr{U}$.)

When $\kappa=\aleph_{0}$, we have property $L$ as defined by Bacon in [2].

THEOREM 1.2. [2] Every space which satisfies property $L$ is isocompact.

REMARK. If $\alpha \leqq \beta \in$ Card and $X$ satisfies property $\beta L$, then $X$ satisfies property $\alpha L$.

The following statement is clear from the definition, but we state it here since it implies that within the class of spaces having countable tightness, and thus the class of sequential spaces, we need 
only consider property $L$ in attempting to establish relationships between classes. Recall that a space $X$ is said to have countable tightness provided that whenever $A \subset X$ and $x \in \bar{A}$, then there is a countable set $C \subset A$ with $x \in \bar{C}$

THEOREM 1.3. If $X$ is a space with countable tightness, then for each $\kappa \in$ Card, $X$ satisfies property $\kappa L$ if and only if $X$ satisfies property $L$.

In many cases, the members of a class of spaces satisfy property $\kappa L$ for every $\kappa \in$ Card. We demonstrate this fact for certain well known classes in the next few paragraphs. We see in 1.4 that symmetrizable spaces satisfy property $\kappa L$ for every $\kappa \in$ Card. This is not true of certain weakenings of symmetrizability, see [5], [6].

THEOREM 1.4. [5] Every symmetrizable space satisfies property $\kappa L$ for every $\kappa \in$ Card.

THEOREM 1.5. Every $\delta \theta-r e f i n a b l e ~ s p a c e ~ s a t i s f i e s$ property $\kappa L$ for every $\kappa \in$ Card.

Proof. Suppose $\mathscr{C}$ is an open cover of the $\delta \theta$-refinable space $X$. Let $\bigcup_{n \in \omega} \mathscr{V}_{n}$ be a $\delta \theta$-refinement of $\mathscr{C}$, i.e., for each $n \in \omega, \mathscr{V}_{n}$ is an open refinement of $\mathscr{C}$ and covers $X$, and for each $x \in X$, there is $n_{x} \in \omega$ such that ord $\left(x, \mathscr{V}_{n_{x}}\right) \leqq \aleph_{0}$. For each $V \in \mathbf{U}_{n \in \omega} \mathscr{V}_{n}$, choose $U_{V} \in \mathscr{C}$ with $V \subset U_{V}$. For each $n \in \omega$, we define $\mathscr{D}_{n}=\left\{\{x\}:\right.$ ord $\left(x, \mathscr{V}_{n}\right) \leqq$ $\left.\mathcal{W}_{0}\right\}$ and we define $f_{n}: \mathscr{D}_{n} \rightarrow \omega \mathscr{U}$ by $f_{n}(\{x\})=U\left\{U_{V}: x \in V \in \mathscr{V}_{n}\right\}$. Suppose $\mathscr{G} \subset \mathscr{D}_{n}$, and $x: \mathscr{G} \rightarrow \cup \mathscr{G}$ with $x(G) \in G$ for every $G \in \mathscr{G}$, and let $y \in \overline{\{x(G): G \in \mathscr{C}\}}$. Choose $V \in \mathscr{V}_{n}$ with $y \in V$. Then there exists $G \in \mathscr{G}$ with $x(G) \in V$. Thus $y \in U_{V} \subset f_{n}(G)$, and so we have $\overline{\{x(G): G \in \mathscr{G}\}} \subset \cup f_{n}(\mathscr{G})$. Since no reference is made to $|\mathscr{G}|$, this completes the proof.

It follows from 1.5 that all semistratifiable [4] and all regular $\sigma$-spaces [14] satisfy property $\kappa L$ for every $\kappa \in$ Card. We mention these since they are classes which are of interest to many topologists. On the other hand, the ordinal space $\omega_{1}$ does not satisfy property $\kappa L$ for any $\kappa \in$ Card, thus we cannot get an analogue to 1.5 for the $M$-spaces [13], or $p$-spaces [1], for instance.

We see in the next theorem that each of the properties $\kappa L$ for $\kappa \in$ Card is preserved by closed continuous functions.

THEOREM 1.6. If $f: X \rightarrow Y$ is a closed continuous surjection, then for each $\kappa \in C$ Card, if $X$ satisfies property $\kappa L$, then so does $Y$. 
Proof. Suppose $\mathscr{U}$ is an open cover of $Y$. Then $\mathscr{U}^{*}=\left\{f^{-1}(U)\right.$ : $U \in \mathscr{C}\}$ is an open cover of $X$. Since $X$ satisfies property $\kappa L$, we may choose a sequence $\left\langle\mathscr{D}_{n}^{*}: n \in \omega\right\rangle$ of collections of subsets of $X$ such that $\mathrm{U}_{n \in \omega} \mathscr{D}_{n}^{*}$ covers $X$ and, for each $n \in \omega, \mathscr{D}_{n}^{*}$ is $\kappa$-weakly cushioned in $\omega \mathscr{U}^{*}$. For each $n \in \omega$, we let $\mathscr{D}_{n}=\left\{f\left(D^{*}\right): D^{*} \in \mathscr{D}_{n}^{*}\right\}$. Since $f$ is surjective, $\bigcup_{n \in \omega} \mathscr{D}_{n}$ covers $Y$. Suppose $n \in \omega$. For each $D \in \mathscr{D}_{n}$, pick $A_{D} \in \mathscr{D}_{n}^{*}$ such that $D=f\left(A_{D}\right)$. Suppose $\phi_{n}^{*}: \mathscr{D}_{n}^{*} \rightarrow \omega \mathscr{C}^{*}$ is the function by which $\mathscr{D}_{n}^{*}$ is $\kappa$-weakly cushioned in $\omega^{*}$. We define $\phi_{n}: \mathscr{D}_{n} \rightarrow \omega \mathscr{U}$ by $\phi_{n}(D)=f\left(\phi_{n}^{*}\left(A_{D}\right)\right)$ for each $D \in \mathscr{D}_{n}$. Suppose $n \in \omega$, $\mathscr{G} \subset \mathscr{D}_{n}$ with $|\mathscr{G}| \leqq \kappa$, and $y: \mathscr{G} \rightarrow \cup \mathscr{G}$ such that $y(G) \in G$ for every $G \in \mathscr{G}$. For each $G \in \mathscr{G}$ pick $x(G) \in A_{G}$ such that $f(x(G))=y(G)$. Then

$$
\begin{aligned}
\overline{\{y(G): G \in \mathscr{G}\}} & =\overline{\{f(x(G)): G \in \mathscr{G}}\} \\
& =\overline{f(\{x(G): G \in \mathscr{C}\}}) \\
& =f(\overline{\{x(G): G \in \mathscr{C}\}}) \\
& \subset f\left(\cup \phi_{n}^{*}\left(\left\{A_{G}: G \in \mathscr{C}\right\}\right)\right) \\
& =f\left(\cup\left\{\phi_{n}^{*}\left(A_{G}\right): G \in \mathscr{C}\right\}\right) \\
& =\cup\left\{f\left(\phi_{n}^{*}\left(A_{G}\right)\right): G \in \mathscr{C}\right\} \\
& =\cup\left\{\phi_{n}(G): G \in \mathscr{C}\right\} .
\end{aligned}
$$

The third equality holds since $f$ is a closed continuous function. The inclusion holds since $\left|\left\{A_{G}: G \in \mathscr{G}\right\}\right| \leqq|\mathscr{G}| \leqq \kappa$. The other relations are obvious, and the theorem is proved.

We see from the next theorem that each of the properties $\kappa L$ for $\kappa \in$ Card is preserved by perfect preimages. Thus, for perfect mappings, we have property $\kappa L$ preserved in both directions, in view of 1.6 .

THEOREM 1.7. If $f: X \rightarrow Y$ is a closed continuous surjection and, for each $y \in Y, f^{-1}(\{y\})$ is Lindelöf, then for each $\kappa \in$ Card, if $Y$ satisfies property $\kappa L$, then so does $X$.

Proof. Let $\mathscr{C}$ be an open cover of $X$. For each $y \in Y$, there is an element $U$ of $\omega \mathscr{Q}$ which contains $f^{-1}(\{y\})$. Since $f$ is a closed continuous function, there exists an open subset $V$ of $Y$ containing $y$ such that $f^{-1}(V) \subset U$. Thus we have an open refinement $\mathscr{W}$ of $\omega \mathscr{C}$ of the form $\mathscr{W}=\left\{f^{-1}(V): V \in \mathscr{V}\right\}$, where $\mathscr{V}$ is an open cover of $Y$. Apply property $\kappa L$ to $\mathscr{V}$ to obtain sequences $\left\langle\mathscr{D}_{n}: n \in \omega\right\rangle$ and $\left\langle\phi_{n}: n \in \omega\right\rangle$ with $\mathscr{D}_{n} \kappa$-weakly cushioned in $\omega \mathscr{\mathscr { V }}$ by $\phi_{n}$ for each $n \in \omega$. For each $n \in \omega$, we let $\mathscr{H}_{n}=\left\{f^{-1}(D): D \in \mathscr{D}_{n}\right\}$ and define $\psi_{n}: \mathscr{H}_{n} \rightarrow \omega \mathscr{W}$ by $\psi_{n}\left(f^{-1}(D)\right)=f^{-1}\left(\phi_{n}(D)\right)$. Clearly, $\bigcup_{n \in \omega} \mathscr{C}_{n}$ covers $X$. We shall show that for each $n \in \omega, \mathscr{H}_{n}$ is $\kappa$-weakly cushioned in $\omega \mathscr{W}$ and 
thus in $\omega \mathscr{Z}$. Suppose $\mathscr{G} \subset \mathscr{H}_{n}$ with $|\mathscr{G}| \leqq \kappa$. Then there exists $\mathscr{E} \subset \mathscr{D}_{n}$ with $|\mathscr{E}| \leqq \kappa$ such that $\mathscr{G}=\left\{f^{-1}(D): D \in \mathscr{E}\right\}$. Suppose $x: \mathscr{G} \rightarrow \cup \mathscr{C}$ with $x(G) \in G$ for each $G \in \mathscr{G}$ and $z \in \overline{\{x(G): G \in \mathscr{C}\}}$. Since $z \in \overline{\left\{x\left(f^{-1}(D)\right): D \in \mathscr{E}\right\}}$, we have that $f(z) \in\left\{\overline{\left.f\left(x\left(f^{-1}(D)\right)\right): D \in \mathscr{E}\right\}} \subset \cup \phi_{n}(\mathscr{E})\right.$. Thus $z \in f^{-1}\left(\cup \dot{\phi}_{n}(\mathscr{E})\right)=\cup\left\{f^{-1}\left(\dot{\phi}_{n}(D)\right): D \in \mathscr{E}\right\}=\cup \boldsymbol{\psi}_{n}(\mathscr{C})$. This completes the proof.

CoRollaRY 1.7.1. For every $\kappa \in$ Card, property $\kappa L$ is preserved by perfect preimages.

2. Property $\theta L$. The similarity between the type of results which one may obtain using property $L$ and weak $\delta \theta$-refinability (see [5], [17]) leads one to believe that there must be some relationship between these properties. However, Examples 3.3 and 3.4 of [6] are weakly $\delta \theta$-refinable (in fact, weakly $\bar{\theta}$-refinable [15]) spaces which do not satisfy property $L$. Further, for each $\kappa \in$ Card, the set $\kappa^{+}$with the topology generated by the subsets whose compliment's cardinality does not exceed $\kappa$, i.e., the co- $\kappa$ topology, is a space which satisfies property $\kappa L$ and is not $\delta \theta$-refinable. Gary Gruenhage has recently constructed an example, assuming the continuum hypothesis, of a space with a point countable base (hence, satisfying property $\kappa L$ for every $\kappa \in$ Card) which is not weakly $\theta$ refinable. At this time, no example is known to the author of a space which satisfies property $L$ and is not weakly $\delta \theta$-refinable. It seems that such a space must surely exist however.

We now define and discuss a property which generalizes both property $L$ and weak $\delta \theta$-refinability.

Definition 2.1. For $\kappa \in$ Card, we say a space $X$ satisfies property $\theta \kappa L$ if and only if for every open cover $\mathscr{C}$ of $X$ there exists a sequence $\left\langle\mathscr{D}_{n}: n \in \omega\right\rangle$ of collections of subsets of $X$ and a sequence $\left\langle\mathscr{V}_{n}: n \in \omega\right\rangle$ of open refinements of $\mathscr{C}$ such that $\bigcup_{n \in \omega} \mathscr{D}_{n}$ covers $X$ and for each $n \in \omega, \cup \mathscr{D}_{n} \subset \cup \mathscr{\mathscr { V }}_{n}$ and $\mathscr{D}_{n}$ is $\kappa$-weakly cushioned in $\omega \mathscr{V}_{n}$ in the space $\cup \mathscr{\mathscr { V }}_{n}$.

In analogy with the treatment of property $\aleph_{0} L$, we shall refer to property $\theta \aleph_{0} L$ by property $\theta L$. No confusion will arise between the notations $\theta L$ and $\kappa L$ since we shall never use the symbol $\theta$ to describe the cardinality of a collection.

Below we list a few results which are immediate from the definitions of these properties.

Proposition 2.1.1.

(a) For each $\kappa \in$ Card, if $X$ satisfies property $\theta \kappa L$ and $A$ is an $F_{\sigma}$-subset of $X$, then $A$ satisfies property $\theta \kappa L$. 
(b) For each $\kappa \in C a r d$, if $X$ has countable tightness, then $X$ satisfies property $\theta \kappa L$ if and only if $X$ satisfies property $\theta L$.

(c) If $\alpha, \beta \in$ Card and $\alpha \leqq \beta$, then any space which satisfies property $\theta \beta L$ also satisfies property $\theta \alpha L$.

THEOREM 2.2. Every weakly ôt-refinable space satisfies property $\theta \kappa L$, for every $\kappa \in$ Card.

Proof. Suppose $X$ is a weakly $\delta \theta$-refinable space and $\mathscr{C}$ is an open cover of $X$. Let $\bigcup_{n \in \omega} \mathscr{V}_{n}$ be a weak $\delta \theta$-refinement of $\mathscr{C}$, i.e., $\bigcup_{n \in \omega} \mathscr{Y}_{n}$ is an open refinement of $\mathscr{C}$ which covers $X$ and for each $x \in X$, there is $n_{x} \in \omega$ such that $0<$ ord $\left(x, \mathscr{\mathscr { N }}_{n_{x}}\right) \leqq \boldsymbol{\aleph}_{0}$. Let $\mathscr{D}_{n}=$ $\left\{\{x\}: 0<\operatorname{ord}\left(x, \mathscr{V}_{n}\right) \leqq \boldsymbol{\aleph}_{0}\right\}$, for each $n \in \omega$. Define $f_{n}: \mathscr{D}_{n} \rightarrow \omega \mathscr{V}_{n}$ by $f_{n}(\{x\})=\cup\left\{V: x \in V \in \mathscr{C}_{n}\right\}$, for each $n \in \omega$. Suppose $\mathscr{C} \subset \mathscr{D}_{n}, x: \mathscr{G} \rightarrow \cup \mathscr{G}$ with $x(G) \in G$ for each $G \in \mathscr{G}$, and $z \in \overline{\{x(G): G \in \mathscr{G}\}} \cap \cup \mathscr{V}_{n}$. Since $z \in \cup \mathscr{T}_{n}$, we may choose $V \in \mathscr{Y}_{n}$ with $z \in V$. Then there exists $G \in \mathscr{G}$ such that $x(G) \in V$. Thus $z \in V \subset f_{n}(\{x(G)\})$. Since $|\mathscr{G}|$ is not considered in the above argument, we have that $\mathscr{D}_{n}$ is $\kappa$-weakly cushioned in $\omega \mathscr{\mathscr { V }}_{n}$ in the space $\cup \mathscr{V}_{n}$, for every $\kappa \in$ Card.

THEOREM 2.3. For each $\kappa \in$ Card, every space which satisfies property $\kappa L$ also satisfies property $\theta \kappa L$.

Proof. If $X$ satisfies property $\kappa L$ and $\mathscr{C}$ is an open cover of $X$, then we let the $\mathscr{V}_{n}$ in the definition of property $\theta \kappa L$ be $\mathscr{C}$ for each $n \in \omega$. The theorem clearly follows.

THEOREM 2.4. Every space which satisfies property $\theta L$ is isocompact.

Proof. Since property $\theta L$ is clearly closed hereditary, we need only show that every countably compact space which satisfies property $\theta L$ is compact.

Suppose $X$ is a space which satisfies property $\theta L$ and $\mathscr{U}$ is an open cover of $X$. Let $C(\mathscr{Z})$ be the collection of all subsets of $X$ which are covered by a countable subcollection of $\mathscr{U}$. Apply the definition of property $\theta L$ to $\mathscr{C}$ to obtain sequences $\left\langle\mathscr{D}_{n}: n \in \omega\right\rangle$, $\left\langle\mathscr{V}_{n}: n \in \omega\right\rangle$ and $\left\langle f_{n}: n \in \omega\right\rangle$ such that, for each $n \in \omega, \mathscr{V}_{n}$ is an open refinement of $\mathscr{H}, \cup \mathscr{D}_{n} \subset \cup \mathscr{\mathscr { V }}_{n}$, and $\mathscr{D}_{n}$ is a collection of subsets of $X$ which is $\aleph_{0}$-weakly cushioned in $\omega \mathscr{Y}_{n}$ is the space $\cup \mathscr{\mathscr { V }}_{n}$ by the function $f_{n}: \mathscr{D}_{n} \rightarrow \omega \mathscr{\mathscr { V }}_{n}$, and $\bigcup_{n \in \omega} \mathscr{D}_{n}$ covers $X$. Let $C_{n}=\cup \mathscr{D}_{n}$ for each $n \in \omega$. If $X \in C(\mathscr{U})$, then $\mathscr{C}$ contains a finite subcover. Therefore, we assume $X \notin C(\mathscr{C})$. We define $n_{0}$ to be the first element of the set $\left\{m: C_{m} \notin C(\mathscr{C})\right\}$. Let $E_{0}=X \backslash \cup \mathscr{C}$, where $\mathscr{C} \subset \mathscr{Q}_{\mathscr{L}}$ is countable 
and covers $C_{j}$ for each $j<n_{0}$. Suppose $k<\omega$ and $E_{j}, n_{j}$ are chosen for $j \leqq k$ such that:

(1) $E_{j}$ is closed.

(2) $n_{j}=$ first element of the set $\left\{m: E_{j} \cap C_{m} \notin C(\mathscr{C})\right\}$.

(3) $E_{j+1} \subset E_{j}$, whenever $E_{j+1}$ is defined.

(4) $E_{j} \cap C_{m}=\varnothing$, for $m<n_{j}$.

Let $B=E_{k} \backslash \cup \mathscr{O}_{n_{k}}$. We claim that $B \notin C(\mathscr{C})$. Suppose $\mathscr{H} \subset \mathscr{Z}$ is countable and $B \subset \cup \mathscr{C}$. Since $E_{k} \cap C_{n_{k}} \notin C(\mathscr{C})$, it follows that there exists $a_{0} \in\left(E_{k} \cap C_{n_{k}}\right) \backslash \cup \mathscr{K}$. Choose $D_{0} \in \mathscr{D}_{n_{k}}$ such that $a_{0} \in D_{0}$. Let $U_{0}=f_{n_{k}}\left(D_{0}\right)$. Suppose $m<\omega$ and $a_{j}, D_{j}, U_{j}$ are chosen for $j \leqq m$ such that:
(i) $a_{j} \in D_{j} \in \mathscr{D}_{n_{k}}$
(ii) $a_{j} \in\left(E_{k} \cap C_{n_{k}}\right) \backslash\left(\mathbf{U}_{s<j} f_{n_{k}}\left(D_{s}\right) \cup \cup \mathscr{C}\right)$.
(iii) $U_{j}=f_{n_{k}}\left(D_{j}\right) \backslash\left\{a_{s}: s<j\right\}$.

The set $\bigcup_{j \leqq m} f_{n_{k}}\left(D_{j}\right)$ is the union of a countable subcollection of $\mathscr{V}_{n_{k}}$. Hence there exists a countable subcollection $\mathscr{W}$ of $\mathscr{C}$ such that $\bigcup_{j \leqq m} f_{n_{k}}\left(D_{j}\right) \cup \cup \mathscr{X} \subset \cup \mathscr{W}$. By (2) above, there exists $a_{m+1} \in$ $\left(E_{k} \cap C_{n_{k}}\right) \backslash U \mathscr{Y}$. Choose $D_{m+1} \in \mathscr{D}_{n_{k}}$ such that $a_{m+1} \in D_{m+1}$ and let $U_{m+1}=$ $f_{n_{k}}\left(D_{m+1}\right) \backslash\left\{a_{j}: j \leqq m\right\}$. In this way we construct $\left\{a_{m}: m \in \omega\right\},\left\{U_{m}: m \in \omega\right\}$ and $\left\{D_{m}: m \in \omega\right\}$. Suppose $x \in \overline{\left\{a_{m}: m \in \omega\right\}}$. By the construction, $x \notin \cup \mathscr{C}$, but $x \in E_{k}$ since $E_{k}$ is closed. Thus $x \in\left(E_{k} \backslash \cup \mathscr{C}\right) \subset \cup \mathscr{V}_{n_{k}}$, and so $x \in \overline{\left\{a_{m}: m \in \omega\right\}} \cap \cup \mathscr{\mathscr { N }}_{n_{k}}$. Now $\mathscr{D}_{n_{k}}$ is $\aleph_{0}$-weakly cushioned in $\omega \mathscr{V}_{n_{k}}$, in the space $\cup \mathscr{\mathscr { V }}_{n_{k}}$; thus, $x \in \mathrm{U}_{m \in \omega} f_{n_{k}}\left(D_{m}\right)$. It follows from the $\aleph_{0}$-weak cushioning of $\mathscr{D}_{n_{k}}$ in $\omega \mathscr{V}_{n_{k}}$ that $\left\{U_{m}: m \in \omega\right\}$ is a countable open cover of $\overline{\left\{a_{m}: m \in \omega\right\}}$ with no finite subcover. Of course, $\overline{\left\{a_{m}: m \in \omega\right\}}$ is a closed subset of $X$; hence, it is countably compact. This contradiction establishes that $B \notin C(\mathscr{Z})$. There exists $m \in \omega$ such that $B \cap C_{m} \notin C(\mathscr{C})$. Let $n_{k+1}$ be the first such $m$. Let $\mathscr{W}$ be a countable subcollection of $\mathscr{C}$ covering $B \cap C_{j}$ for each $j<n_{k+1}$. Let $E_{k+1}=B \backslash \cup \mathscr{W}$. The set $E_{k+1}=B \backslash \cup \mathscr{W}=\left(E_{k} \backslash \cup \mathscr{\mathscr { N }}_{n_{k}}\right) \backslash \cup \mathscr{W}$ is clearly closed. By choice of $n_{k+1}$ and by the observation above, (2) and (3) are satisfied. Since $\left(\bigcup_{j<n_{k+1}} C_{j}\right) \cap B \subset \cup \mathscr{V}$, condition (4) is satisfied. Note that $n_{k+1}>n_{k}$, by construction, and thus, by (4), $\cap\left\{E_{k}: k \in \omega\right\}=\varnothing$. However, $\left\langle E_{k}: k \in \omega\right\rangle$ is decreasing sequence of nonempty closed sets. This contradiction to the countable compactness of $X$ establishes the result.

The above proof combines the ideas of the proof of the lemma in [5] with the proof in [16] of the isocompactness of weakly $\delta \theta$ refinable spaces. This style of proof can be used to obtain certain strengthenings of theorems of Blair [3] as we see in the following three theorems.

Recall that a space is called closed-complete [9] (respectively, realcompact [10]) in case every closed (respectively, $z$-) ultrafilter with the countable intersection property is fixed. (In addition, real- 
compact spaces are required to be completely regular and $T_{2}$.)

THEOREM 2.5. If $X$ is a $T_{1} \boldsymbol{\aleph}_{1}$-compact space which satisfies property $\theta \mathbf{\aleph}_{1} L$, then $X$ is closed-complete.

Proof. Suppose is a free closed ultrafilter on $X$ which has the countable intersection property. Let $\mathscr{C}=\{X \backslash F: F \in \mathscr{F}\}$. The collection $\mathscr{Z} 6$ is an open cover of $X$ with no countable subcover. As we did in the proof of 2.4 , we shall construct a sequence $\left\langle E_{k}: k \in \omega\right\rangle$ satisfying (1)-(4) of the proof of 2.4. Our proof here will differ (in the notation of the proof of 2.4) in the way we show that $B \in C\left(\varpi_{6}\right)$. To see that this is true, we continue the construction used in the proof of 2.4 to obtain sets $\left\{a_{\alpha}: \alpha<\omega_{1}\right\},\left\{U_{\alpha}: \alpha<\omega_{1}\right\}$, and $\left\{D_{n}: \alpha<\omega_{1}\right\}$ such that:

(i) For each $\alpha<\omega_{1}, a_{\alpha} \in D_{\alpha} \in \mathscr{D}_{n_{k}}$.

(ii) For each $\alpha<\omega_{1}, a_{\alpha} \in\left(E_{k_{k}} \cap C_{n_{k}}\right) \backslash\left(\bigcup_{\beta<\alpha} f_{n_{k}}\left(D_{\beta}\right) \cup \cup \mathscr{C}\right)$.

(iii) For each $\alpha<\omega_{1}, U_{\kappa}=f_{n_{k}}\left(D_{\alpha}\right) \backslash \overline{\left\{a_{\beta}: \beta<\alpha\right\}}$. Reasoning similar to that used in the proof of 2.4 shows that $\left\{U_{n}: \alpha<\omega_{1}\right\}$ is an open cover of $\overline{\left\{a_{\alpha}: \alpha<\omega_{1}\right\}}$, but for each $\beta<\omega_{1}$, $U_{\beta} \cap\left\{a_{\alpha}: \alpha<\omega_{1}\right\}=\left\{a_{\beta}\right\}$ which contradicts the $\aleph_{1}$-compactness of $X$. For each $k \in \omega$, no countable subcollection of $\not{Z}$ covers $E_{k}$, and thus $E_{k} \cap F \neq \varnothing$ for each $F \in \mathscr{F}$. However, $\mathscr{F}$ is a closed ultrafilter, and so $E_{k} \in \mathscr{F}$ for every $k \in \omega$. But $\cap\left\{E_{k}: k \in \omega\right\}=\varnothing$ contradicting the countable intersection property and the result is established.

From the proof above and the proof of 2.2, we have the following analogue of Corollary 3.2 of [3].

COROLlaRy 2.5.1. If every uncountable discrete subset of $X$ has a 2-limit point in $X$, then the following are equivalent:

(a) $X$ is closed complete.

(b) If $\mathscr{F}^{-}$is any free closed ultrafilter on $X$, then we may apply $\theta \aleph_{1} L$ to $\{X \backslash F: F \in \mathscr{T}\}$. (In saying "we may apply $\theta \aleph_{1} L$ to $\{X \backslash F: F \in \mathscr{F}\}$ ", we mean that if we let $\mathscr{C}=\{X \backslash F: F \in \mathscr{F}\}$ then we may obtain sequences $\left\langle\mathscr{D}_{n}: n \in \omega\right\rangle$ and $\left\langle\mathscr{C}_{n}: n \in \omega\right\rangle$ as in 2.1 with $\kappa=\boldsymbol{\aleph}_{1}$.)

THEOREM 2.6. If $X$ is an $\aleph_{1}$-compact $T_{1}$ closed subspace of a product of regular spaces which satisfy property $\theta{ }_{1} L$, then $X$ is closed-complete.

Proof. This can be established exactly as 3.4 of [3], since inverse projection mappings preserve weak cushioning.

A space $X$ is a cb-space [11] provided for each decreasing sequence $\left\langle F_{n}: n \in \omega\right\rangle$ of closed subsets of $X$ with $\bigcap_{n \in \omega} F_{n}=\varnothing$ there 
is a sequence $\left\langle Z_{n}: n \in \omega\right\rangle$ of zero-sets of $X$ such that $Z_{n} \supset F_{n}$ for each $n \in \omega$ and $\bigcap_{n \in \omega} Z_{n}=\varnothing$.

THEOREM 2.7. If $X$ is a $T_{2}$ completely regular $\aleph_{1}$-compact cbspace, then the following are equivalent.

(a) $X$ is realcompact.

(b) $X$ can be embedded as a closed subspace of a product of regular weakly $\delta \theta$-refinable spaces.

(c) If $\mathscr{F}$ is any free closed ultrafilter on $X$, then $\{X \backslash F: F \in \mathscr{F}\}$ has a weak $\delta \theta$-refinement.

(d) $X$ can be embedded as a closed subspace of a product of regular spaces which satisfy property $\theta \aleph_{1} L$.

(e) If $\mathscr{F}$ is any free closed ultrafilter on $X$, then we may apply $\theta \aleph_{1} L$ to $\{X \backslash F: F \in \mathscr{F}\}$.

If, in addition, $X$ is normal, then (a), (b), (c), (d), (e) are also equivalent to (f) and (g).

(f) If $\mathscr{A}$ is any free $z$-ultrafilter on $X$, then $\{X \backslash Z: Z \in \mathscr{A}\}$ has a weak $\delta \theta$-refinement.

(g) If $\mathscr{A}$ is any free $z$-ultrafilter on $X$, then we may apply $\theta \aleph_{1} L$ to $\{X \backslash Z: Z \in \mathscr{A}\}$.

Proof. The equivalence of (a), (b), (c) and, for normal $X$, (f) is due to Blair [3]. It is clear that (c) implies (e), (b) implies (d) and (f) implies (g). The fact that (d) implies (a) and (e) implies (a) follows from 2.5, 2.6 and the result of Dykes [9] that $a T_{2}$ completely regular closed-complete $c b$-space is realcompact. The fact that, for normal spaces, (g) implies (e) can be proved in exactly the same manner as Blair's proof that (f) implies (c). Hence the theorem is established.

As we saw in 2.1.1, we may replace " $\theta{ }_{1} L$ " in the above with " $\theta L$ " under the additional assumption that the spaces with which we are working have countable tightness.

Within the framework of isocompact spaces, it has been a question of considerable interest whether $\aleph_{1}$-compactness will imply the Lindelof property, [15], [8], [5]. We see in [5] that the answer is "yes" for $T_{1}$ spaces satisfying property $\aleph_{1} L$, and hence for $T_{1}$ spaces with countable tightness satisfying property $L$. Let us now consider similar results for spaces satisfying property $\theta \kappa L$. We will not have so nice a theorem as was obtained for property $L$, of course, since the example given in [8] is weakly $\theta$-refinable (hence, satisfies property $\theta \kappa L$ for every $\kappa \in$ Card by 2.2$), \aleph_{1}$-compact and not Lindelöf. We do obtain several results by assuming that certain of the conditions are satisfied hereditarily, or by assuming that closed subsets are $G_{i}$-sets. Analogues of 2.8 and 2.10 have previously been obtained 
for weakly $\delta \theta$-refinable spaces. These results were communicated to the author by $\mathrm{H}$. H. Wicke.

THEOREM 2.8. If $X$ satisfies property $\theta L$ and is hereditarily $\aleph_{1}$-compact, then $X$ is Lindelöf.

Proof. Suppose $\mathscr{C}$ is an open cover of $X$ with no countable subcover. Apply the definition of property $\theta L$ to obtain $\left\langle\mathscr{D}_{n}: n \in \omega\right\rangle$ and $\left\langle\mathscr{V}_{n}: n \in \omega\right\rangle$ as in 2.1 with $\kappa=\mathcal{\aleph}_{0}$. There exists $n \in \omega$ such that no countable subcollection of $\mathscr{\mathscr { V }}_{n}$ covers $\cup \mathscr{D}_{n}$. We apply the lemma in [5] in the space $\cup \mathscr{\mathscr { V }}_{n}$ to obtain functions $a: \omega_{1} \rightarrow \cup \mathscr{D}_{n}$, $D: \omega_{1} \rightarrow \mathscr{D}_{n}$ and $U: \omega_{1} \rightarrow$ the open subsets of $\left.\cup \mathscr{\mathscr { V }}_{n}\right\}$ such that the following are true:

(1) For each $\alpha \in \omega_{1}, a_{\alpha} \in \cup \mathscr{D}_{n} \backslash \bigcup_{\beta<\alpha} f_{n}\left(D_{\beta}\right)$.

(2) For each $\alpha \in \omega_{1} a_{\alpha} \in D_{\alpha}$.

(3) For each $\alpha \in \omega_{1} U_{\alpha} \cap\left\{a_{\beta}: \beta \in \omega_{1}\right\}=\left\{a_{\alpha}\right\}$.

(4) $\mathrm{U}_{\alpha \in \omega_{1}} \mathrm{U}_{\alpha}=U_{\alpha \in \omega_{1}} f_{n}\left(D_{\alpha}\right)$.

(5) $\left|\left\{a_{\alpha}: \alpha<\omega_{1}\right\}\right|=\aleph_{1}$.

( $f_{n}$ is the cushioning function for the $n$th level.) The set $\left\{a_{\alpha}: \alpha<\omega_{1}\right\}$ is uncountable and relatively discrete. This contradiction establishes the result.

COROLlARY 2.8.1. A space is hereditarily Lindelöf if and only if it is hereditarily $\aleph_{1}$-compact and hereditarily satisfies property $\theta L$.

TheORem 2.9. For each $\kappa \in$ Card, if $X$ is perfect and satisfies property $\theta \kappa L$, then $X$ satisfies property $\kappa L$.

Proof. Suppose $\mathscr{C}$ is an open cover of $X$. Apply the definition of property $\theta \kappa L$ to obtain sequences $\left\langle\mathscr{D}_{n}: n \in \omega\right\rangle$ and $\left\langle\mathscr{\mathscr { V }}_{n}: n \in \omega\right\rangle$ as in 2.1. Note that we may assume the collection $\mathscr{D}_{n}$ to be a disjoint collection, for each $n \in \omega$. For each $n \in \omega$, choose a sequence $\langle F(n, k): k \in \omega\rangle$ of closed sets such that $U \mathscr{V}_{n}=\bigcup_{k \in \omega} F(n, k)$. We define $\mathscr{D}_{(n, k)}^{*}=\left\{D \cap F(n, k): D \in \mathscr{D}_{n}\right\}$. For each $(n, k) \in \omega \times \omega, F(n, k)$ is a closed set which is contained in $U \mathscr{V}_{n}$. Since $\mathscr{D}_{n}$ is $\kappa$-weakly cushioned in $\omega \mathscr{V}_{n}$ in the space $\cup \mathscr{V}_{n}$ and $\mathscr{V}_{n}$ refines $\mathscr{C}_{6}$ for each $n \in \omega$, and $\cup \mathscr{D}_{(n, k)}^{*} \subset \cup \mathscr{D}_{n} \cap F(n, k)$ for each $(n, k) \in \omega \times \omega$, we easily see that $\mathscr{D}_{(n k)}^{*}$ is $\kappa$-weakly cushioned in $\omega \mathscr{C}$ for each $(n, k) \in \omega \times \omega$ by the cushioning function $f_{(n, k)}^{*}$ defined in the obvious way from $f_{n}$, and the theorem follows.

We can use 2.9 together with 3.6 of [5] to obtain the following results. These could also be obtained from 2.8 using 2.1.1a. 
TheOREM 2.10. If $X$ is a perfect, $T_{1}, \aleph_{1}$-compact space which satisfies property $\theta L$, then $X$ is hereditarily Lindelöf.

CoRollary 2.10.1. If $X$ is a $T_{3}$ space, then $X$ is hereditarily Lindelöf if and only if $X$ is perfect, $\aleph_{1}$-compact and satisfies property $\theta L$.

The following can be proved in the same way that we proved 1.7. Unfortunately, we do not know if the analogue of 1.6 holds for property $\theta \kappa L$.

THEOREM 2.11. If $f: X \rightarrow Y$ is a closed continuous surjection and, for each $y \in Y, f^{-1}(\{y\})$ is Lindelöf, then for each $\kappa \in$ Card, if $Y$ satisfies property $\theta \kappa L$, then so does $X$.

CoRollaRy 2.11.1. For each $\kappa \in$ Card, property $\theta \kappa L$ is preserved under perfect preimages.

Examples are readily available to demonstrate that property $\theta L$ is strictly weaker than property $L$. In fact, the examples in [8] and $\Sigma$ in [7] both have property $\theta \kappa L$ for every $\kappa \in$ Card but do not satisfy property $L$. It is inconceivable to the author that property $\theta L$ could imply weak $\delta \theta$-refinability; however, at this writing no example is known. Of course, an example of a space which satisfies property $L$ and is not weakly $\delta \theta$-refinable would supply the needed example here as well.

3. Questions. The following is a list of some open questions regarding the properties discussed in this paper.

Question 3.1. Is the closed continuous image of a weakly $\delta \theta$ refinable space necessarily weakly $\delta \theta$-refinable?

Question 3.2. Does the closed continuous image of a space which satisfies property $\theta L$ necessarily satisfy property $\theta L$ ?

Question 3.3. Is there a $T_{1}, \aleph_{1}$-compact space which satisfies property $L$ and is not Lindelöf?

Of course, such a space could not satisfy property $\aleph_{1} L$, and therefore could not have countable tightness. Also, such a space could not be hereditarily $\boldsymbol{W}_{1}$-compact, and therefore could not be perfect.

Question 3.4. Is there a $T_{1}, \aleph_{1}$-compact space which satisfies property $\theta L$ (or $L$ ) and is not closed-complete? 
Such a space could not have countable tightness or satisfy property $\theta \aleph_{1} L$.

Question 3.5. Is there a space which satisfies property $L$ (preferably with countable tightness) and is not weakly $\delta \theta$-refinable?

\section{REFERENCES}

1. A. V. Arhangel'skii, Mappings and spaces, Russian Math. Surveys, 21 (1966), 115-162.

2. Philip Bacon, The compactness of countably compact spaces, Pacific J. Math., 32 (1970), 587-592.

3. R. L. Blair, Closed-completeness in spaces with weak covering properties, pp. 17-45 in: Set Theoretic Topology, ed. G. M. Reed, Academic Press, New York, San Francisco, and London, 1977.

4. G. Creede, Semi-stratifiable spaces, Topology Conference, Arizona State University, (1967), 318-323.

5. S. W. Davis, On certain isocompact spaces, Topology Proceedings, 1 (1976), 33-45.

6. —_ On $\mathscr{F}_{r}$-spaces, General Topology and Appl., 9 (1978), 131-138.

7. E. K. van Douwen, A technique for constructing honest locally compact submetrizable examples, to appear.

8. E. K. van Douwen and H. H. Wicke, A real, weird topology on the reals, Houston J. Math., 3 (1977), 141-152.

9. N. Dykes, Generalizations of realcompact spaces, Pacific J. Math., 33 (1970), 571-581. 10. L. Gillman and M. Jerison, Rings of Continuous Functions, Van Nostrand, Princeton, 1960.

11. J. Mack, On a class of countably paracompact spaces, Proc. Amer. Math. Soc., 16 (1965), 467-472.

12. E. Michael, Yet another note on paracompact spaces, Proc. Amer. Math. Soc., 10 (1959), 309-314.

13. K. Morita, Products of normal spaces with metric spaces, Math. Ann., 154 (1964), 365-382.

14. A. Okuyama, $\sigma$-spaces and closed mappings, I, Proc. Japan Acad., 44 (1968), 472-477.

15. J. C. Smith, Properties of weak $\bar{\theta}$-refinable spaces, Proc. Amer. Math. Soc., 53 (1975), 511-517.

16. H. H. Wicke and J. M. Worrell, Jr., Point-countability and compactness, Proc. Amer. Math. Soc., 55 (1976), 427-431.

17. - The hereditary Lindelöf property, primitive structures, and separable metrizability, Notices Amer. Math. Soc., 22 (1975), A-425.

Received December 15, 1976 and in revised form May 13, 1978.

OHIO UNIVERSITY

Athens, OH

AND

AUBURN UNIVERSITY

AUBURN, AL 36830

Current address: Miami University

Oxford, OH 45056 


\section{PACIFIC JOURNAL OF MATHEMATICS}

\section{EDITORS}

RICHARD ARENS (Managing Editor)

University of California

Los Angeles, CA 90024

Charles W. Curtis

University of Oregon

Eugene, OR 97403

C. C. MOORE

University of California

Berkeley, CA 94720
J. DUGUNDJI

Department of Mathematics

University of Southern California

Los Angeles, CA 90007

R. FINN and J. MILGRAM

Stanford University

Stanford, CA 94305

\section{ASSOCIATE EDITORS}

\section{E. F. BeCKENBACH}

B. H. NeumanN

F. WOLF

K. YoSHIDA

\section{SUPPORTING INSTITUTIONS}

\author{
UNIVERSITY OF SOUTHERN CALIFORNIA \\ STANFORD UNIVERSITY \\ UNIVERSITY OF HAWAII \\ UNIVERSITY OF TOKYO \\ UNIVERSITY OF UTAH \\ WASHINGTON STATE UNIVERSITY \\ UNIVERSITY OF WASHINGTON
}

The Supporting Institutions listed above contribute to the cost of publication of this Journal, but they are not owners or publishers and have no responsibility for its content or policies.

Mathematical papers intended for publication in the Pacific Journal of Mathematics should be in typed form or offset-reproduced, (not dittoed), double spaced with large margins. Please do not use built up fractions in the text of the manuscript. However, you may use them in the displayed equations. Underline Greek letters in red, German in green, and script in blue. The first paragraph or two must be capable of being used separately as a synopsis of the entire paper. Items of the bibliography should not be cited there unless absolutely necessary, in which case they must be identified by author and journal, rather than by item number. Manuscripts, in triplicate, may be sent to any one of the editors. Please classify according to the scheme of Math. Reviews, Index to Vol. 39. All other communications should be addressed to the managing editor, or Elaine Barth, University of California, Los Angeles, California, 90024.

50 reprints to each author are provided free for each article, only if page charges have been substantially paid. Additional copies may be obtained at cost in multiples of 50 .

The Pacific Journal of Mathematics is issued monthly as of January 1966. Regular subscription rate: $\$ 72.00$ a year (6 Vols., 12 issues). Special rate: $\$ 36.00$ a year to individual members of supporting institutions.

Subscriptions, orders for numbers issued in the last three calendar years, and changes of address should be sent to Pacific Journal of Mathematics, P.O. Box 969, Carmel Valley, CA 93924, U.S.A. Older back numbers obtainable from Kraus Periodicals Co., Route 100, Millwood, NY 10546.

PUBLISHED BY PACIFIC JOURNAL OF MATHEMATICS, A NON-PROFIT CORPORATION

Printed at Kokusai Bunken Insatsusha (International Academic Printing Co., Ltd.). 8-8, 3-chome, Takadanobaba, Shinjuku-ku, Tokyo 160, Japan. 


\section{Pacific Journal of Mathematics}

\section{Vol. 80, No. $2 \quad$ October, 1979}

K. Adachi, On the multiplicative Cousin problems for $N^{p}(D) \ldots \ldots \ldots \ldots 297$

Howard Banilower, Isomorphisms and simultaneous extensions in $C(S) \ldots 305$

B. R. Bhonsle and R. A. Prabhu, An inversion formula for a distributional

finite-Hankel-Laplace transformation ................... 313

Douglas S. Bridges, Connectivity properties of metric spaces.......... 325

John Patton Burgess, A selection theorem for group actions ........... 333

Carl Claudius Cowen, Commutants and the operator equations

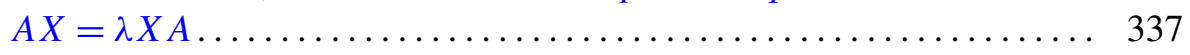

Thomas Curtis Craven, Characterizing reduced Witt rings. II .......... 341

J. Csima, Embedding partial idempotent d-ary quasigroups ............ 351

Sheldon Davis, A cushioning-type weak covering property ............ 359

Micheal Neal Dyer, Nonminimal roots in homotopy trees ............. 371

John Erik Fornaess, Plurisubharmonic defining functions ........... 381

John Fuelberth and James J. Kuzmanovich, On the structure of finitely

generated splitting rings .......................... 389

Irving Leonard Glicksberg, Boundary continuity of some holomorphic

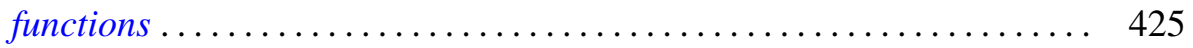

Frank Harary and Robert William Robinson, Generalized Ramsey theory.

IX. Isomorphic factorizations. IV. Isomorphic Ramsey numbers .......

Frank Harary and Allen John Carl Schwenk, The spectral approach to determining the number of walks in a graph...........

David Kent Harrison, Double coset and orbit spaces ..... . .

Shiro Ishikawa, Common fixed points and iteration of commuting

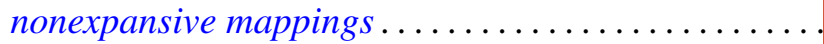

Philip G. Laird, On characterizations of exponential polynomials ........ 503

Y. C. Lee, A Witt's theorem for unimodular lattices ...........

Teck Cheong Lim, On common fixed point sets of commutative

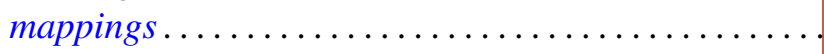

R. S. Pathak, On the Meijer transform of generalized functions ...

T. S. Ravisankar and U. S. Shukla, Structure of $\Gamma$-rings . . .

Olaf von Grudzinski, Examples of solvable and nonsolvable convolution

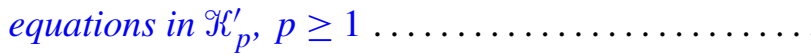

